\title{
Routine Intraoperative Bacterial Culture May Be Needed in Complicated Appendicitis
}

\author{
Jung Tack Son ${ }^{1, \star}$, Gue Chun Lee ${ }^{1, \star}$, Hyung Ook Kim${ }^{1}$, Taewoon Kim ${ }^{1}$, Donghyoun Lee ${ }^{2}$, Sung Ryol Lee ${ }^{1}$, \\ Kyung Uk Jung ${ }^{1}$, Hungdai Kim ${ }^{1}$, Ho-Kyung Chun ${ }^{1}$ \\ ${ }^{1}$ Department of Surgery, Kangbuk Samsung Hospital, Sungkyunkwan University School of Medicine, Seoul; ${ }^{2}$ Department of Surgery, Jeju \\ National University Hospital, Jeju National University School of Medicine, Jeju, Korea
}

Purpose: Choosing the appropriate antibiotic is important for treatment of complicated appendicitis. However, increasing multidrug resistant bacteria have been a serious problem for successful treatment. This study was designed to identify bacteria isolated from patients with complicated appendicitis and reveal their susceptibilities for antibiotics and their relationship with patient clinical course.

Methods: This study included patients diagnosed with complicated appendicitis and examined the bacterial cultures and antimicrobial susceptibilities of the isolates. Data were retrospectively collected from medical records of Kangbuk Samsung Hospital from January 2008 to February 2018.

Results: The common bacterial species cultured in complicated appendicitis were as follows: Escherichia coli $(\mathrm{n}=113$, 48.9\%), Streptococcus spp. ( $\mathrm{n}=29,12.6 \%)$, Pseudomonas spp. $(\mathrm{n}=23,10.0 \%)$, Bacteriodes spp. $(\mathrm{n}=22,9.5 \%)$, Klebsiella $(\mathrm{n}=11,4.8 \%)$, and Enterococcus spp. $(\mathrm{n}=8,3.5 \%)$. In antibiotics susceptibility testing, the positive rate of extended-spectrum beta lactamase (ESBL) was 9.1\% (21 of 231). The resistance rate to carbapenem was 1.7\% (4 of 231), while that to vancomycin was $0.4 \%$ ( 1 of 231 ). E. coli was $16.8 \%$ ESBL positive (19 of 113 ) and had $22.1 \%$ and $19.5 \%$ resistance rates to cefotaxime and ceftazidime, respectively. Inappropriate empirical antibiotic treatment (IEAT) occurred in 55 cases (31.8\%) and was significantly related with organ/space surgical site infection (SSI) (7 of 55, P=0.005).

Conclusion: The rate of antibiotic resistance organisms was high in community-acquired complicated appendicitis in Koreans. Additionally, IEAT in complicated appendicitis may lead to increased rates of SSI. Routine intraoperative culture in patients with complicated appendicitis may be an effective strategy for appropriate antibiotic regimen.

Keywords: Complicated appendicitis; Bacteriology; Antibiotic resistance; Inappropriate empiric antibiotic treatment; Surgical site infection

\section{INTRODUCTION}

Acute appendicitis is a common disease among patients who

Received: July 17, 2019 • Accepted: November 4, 2019

Correspondence to: Hyung Ook Kim, M.D.

Department of Surgery, Kangbuk Samsung Hospital, Sungkyunkwan

University School of Medicine, 29 Saemunan-ro, Jongno-gu, Seoul 03181,

Korea

Tel: +82-2-2001-8550, Fax: +82-2-2001-2131

E-mail: ho115.kim@samsung.com

ORCID: https://orcid.org/0000-0003-0603-8766

*Jung Tack Son and Gue Chun Lee contributed equally as co-first authors.

(c) 2020 The Korean Society of Coloproctology

This is an open-access article distributed under the terms of the Creative Commons Attribution Non-

Commercial License (https://creativecommons.org/licenses/by-nc/4.0) which permits unrestricted non-

commercial use, distribution, and reproduction in any medium, provided the original work is properly cited. come to the emergency room and requires surgery. The lifetime risk of appendicitis is $8.6 \%$ in males and $6.7 \%$ in females [1]. The standard treatment for acute appendicitis is appendectomy with appropriate antibiotic treatment, and the choice of appropriate antibiotics is especially important in treatment of complicated appendicitis. Antimicrobial treatment involves a delicate balance of optimizing empirical therapy, which has been shown to improve clinical outcomes, while simultaneously reducing unnecessary antimicrobial use [2]. Intraoperative culture has been commonly performed from both the appendix and peritoneal fluid or pus, when present. The spectrum of isolated organisms and their antibiotic susceptibility profiles could help physicians choose appropriate antibiotics and treat intra-abdominal infection more effectively.

Two treatment guidelines about intra-abdominal infection have 
been published. The first was published by the Surgical Infection Society and the Infectious Diseases Society of America (SISIDSA) to diagnose and manage intra-abdominal infections [3]. The second was published by the World Society of Emergency Surgery (WSES) in 2017 [4]. In addition, the WSES published their guidelines for diagnosis and management of acute appendicitis in 2016 [5]. According to the SIS-IDSA guidelines, the antibiotics used for empirical treatment of community-acquired intraabdominal infection should be active against enteric gram-negative aerobic and facultative bacilli and enteric gram-positive streptococci. Depending on the severity of the infection, the recommended antibiotics regimens are ticarcillin-clavulanate, cefoxitin, ertapenem, moxifloxacin, or tigecycline as single-agent therapy or a combination of metronidazole with cefazolin, cefuroxime, ceftriaxone, cefotaxime, levofloxacin, or ciprofloxacin [3]. In noncritically ill patients with community-acquired intra-abdominal infection, the WSES guidelines recommend a similar antibiotic regimen including amoxicillin/clavulanate; ceftriazone with metronidazole; cefotaxime with metronidazole; or ciprofloxacin with metronidazole, moxifloxacin, ertapenem, or tigecycline depending on risk of ESBL-producing bacteria $[4,5]$.

Multidrug resistance (MDR) caused by worldwide overuse of antibiotics is an ongoing issue. Especially, organisms with resistance to broad and extended-spectrum antibiotics can lead to complications in treatment. The most relevant MDR pathogens found in acute appendicitis are Enterobacteriaceae, Staphylococcus aureus, Klebsiella pneumoniae, Acinetobacter baumannii, Pseudomonas aeruginosa (P. aeruginosa), and Enterococci bacteria [6]. Patients with MDR bacteria in acute appendicitis suffered more infectious complications and needed longer hospitalizations than patients with antibiotic susceptible bacteria [6]. Furthermore, the prevalence of antibiotic-resistant $E$. coli isolated in intra-abdominal infection is especially high in the Asia-Pacific region [7].

Therefore, this study was designed to confirm isolated bacteria and their susceptibilities to antibiotics to treat complicated appendicitis in Korea and to reveal bacteria and their susceptibilities affecting the clinical course of patients. Furthermore, we confirmed the effectiveness of current empiric antibiotics and the usefulness of bacterial culture in patients with complicated appendicitis.

\section{METHODS}

\section{Patient selection and data collection}

This study includes patients diagnosed with complicated appendicitis. We examined bacterial cultures and performed antimicrobial susceptibility tests on cultures isolated from these patients. Complicated appendicitis is defined a gangrenous change or perforation of the appendix or a phlegmon/abscess caused by appendicitis and present on abdominopelvic computed tomography (CT) or intraoperative findings and proven microscopically. When suppurative periappendiceal/pelvic fluid collection or abscess was present, suppurative fluid or abscess was collected dur- ing the operation. When suppurative fluid or abscess was not present and only gangrenous change of appendix was present, swabbing of the appendiceal lumen was performed. Bacterial culturing was selectively performed at the discretion of each surgeon. Antimicrobial susceptibility testing was conducted using automated instrument systems with test panels. However, some rare organisms that have no test panel were not tested for antimicrobial susceptibility.

All patients underwent an operation including laparoscopic appendectomy or cecal resection. Patients who initially treated with percutaneous drainage are not included in this study. The initial choice of empirical antibiotics was ceftriaxone (3rd generation cephalosporin) with metronidazole. Data before 2008 were not included in this study because these data do not reflect recent bacteriology, which is continuously changing.

Data were retrospectively collected from the medical records of Kangbuk Samsung Hospital from January 2008 to February 2018. Characteristics of patients including sex, age, American Society of Anesthesiologists (ASA) physical status classification, length of hospital stay, and results of laboratory tests were collected in medical records. Diagnosis, types of operation, and complications in patients were confirmed by CT images, intraoperative findings, and pathologic reports. Diagnoses were classified into 1 of 2 groups: (1) complicated appendicitis without abscess and (2) complicated appendicitis with abscess. Postoperative complications and mortality within 30 days after surgery were investigated.

\section{Definitions}

"Healthcare-associated infection" is defined as an infection acquired during the course of receiving healthcare. It includes not only hospital-acquired infections, but also infections in patients living in nursing facilities, those having recent hospitalization within 90 days, and those using aggressive medical therapies (intravenous therapy, wound dressing) at home and invasive therapies (hemodialysis, chemotherapy, radiotherapy) in outpatient clinics within 30 days of the index infection [8]. "Community-acquired infection" is defined as an infection detected within 48 hours of hospital admission in patients without previous contact with healthcare service [8]. "Inappropriate empirical antibiotic treatment (IEAT)" is defined as antibiotics given before the results of the cultures were known resistance to the pathogen in in vitro [9]. "Superficial surgical site infection (SSI)" is defined as an infection that involves only the skin and subcutaneous tissues of incision, and "organ/space SSI" is defined as an infection that involves any part of the anatomy in organs and spaces other than the incision, which is opened or manipulated during operation.

\section{Ethics statement}

This retrospective study was approved by the Institutional Review Board of Kangbuk Samsung Hospital (KBSMC 2019-04-037). The informed consent of patients was waived because of the retrospective nature of the study. 


\section{Statistical analysis}

Collected data were statistically analyzed by IBM SPSS Statistics ver. 24.0 (IBM Co., Armonk, NY, USA). Results were expressed as mean \pm standard deviations (SDs). Continuous variables were compared using Student t-test or the Mann-Whitney U-test. Categorical variables were analyzed with Fisher exact test and the chisquare test. P-values $<0.05$ were considered statistically significant.

\section{RESULTS}

General patient characteristics are shown in Table 1. In the study period, 187 patients had undergone bacterial culture after being diagnosed complicated appendicitis, and the culture results were negative in 14 patients (7.5\%). The positive rate of bacterial culture in complicated appendicitis was $92.5 \%$, and a total of 173 patients was included in this study. The mean age of the enrolled patients was $44.8 \pm 21.3$ years, and mean length of hospital stay was $8.9 \pm 7.0$ days. Male patients $(n=110,63.6 \%)$ were more common than female patients $(n=63,36.4 \%)$. Included in this study were 135 cases of complicated appendicitis (78.0\%) without abscess and 38 cases of appendicitis (22.0\%) with abscess. All operations were conducted by laparoscopic surgery. In 42 cases (18\%), resection of the cecum was needed because of severe inflammation in the appendiceal stump.

Table 1. Patients characteristics $(\mathrm{n}=173)$

\begin{tabular}{lc}
\hline Characteristic & Value \\
\hline Age (yr) & $44.8 \pm 21.3$ \\
Sex & \\
Female & $63(36.4)$ \\
Male & $110(63.6)$ \\
Diagnosis & \\
Complicated appendicitis without abscess & $135(78.0)$ \\
Complicated appendicitis with abscess & $38(22.0)$ \\
Operation & \\
Laparoscopic appendectomy & $131(75.7)$ \\
Laparoscopic colon resection & $42(24.3)$ \\
Complications & $30(17.3)$ \\
Superficial SSI & $8(4.6)$ \\
Organ/space SSI & $9(5.2)$ \\
lleus & $11(6.4)$ \\
Gastroenteritis & $2(1.2)$ \\
Readmission & $11(6.4)$ \\
Hospital stay (day) & $8.9 \pm 7.0$ \\
Antibiotic treatment (day) & $12.7 \pm 7.4$ \\
\hline
\end{tabular}

Values are presented as mean \pm standard deviation or number (\%). SSI, surgical site infection.
The mean length of intravenous antibiotic treatment was $8.8 \pm$ 4.9 days. And $71.7 \%$ (124 of 173) of patients prescribed oral antibiotics when they discharged. In the result, mean length of total antibiotic treatment was mean $12.7 \pm 7.4$ days.

After surgery, 30 cases of complications were observed. The most common complication was ileus (11, 5.2\%). Superficial SSI and organ/space SSI were observed in 8 (4.6\%) and 9 cases $(5.2 \%)$, respectively. Gastroenteritis was relatively rare $(n=2,1.2 \%)$, and 11 patients required readmission for any related reasons (6.4\%). Perioperative mortality was not observed within the first 30 days after surgery.

Data of cultured organisms are summarized at Table 2. The number of cultured organisms was 231 in 173 patients. Two or more organisms were cultured from 45 patients (19.5\%). Gramnegative bacteria $(n=190,82.3 \%)$ were more common than gram-positive bacteria $(\mathrm{n}=41,17.7 \%)$. The most commonly cultured organism was Escherichia coli $(\mathrm{n}=113,48.9 \%)$, while the second most common organism was $P$. aeruginosa $(n=21,9.1 \%)$. Next were Streptococcus anginosus $(\mathrm{n}=11,4.8 \%)$ and Streptococcus constellatus ( $\mathrm{n}=10,4.3 \%)$ followed by Klebsiella pneumonia $(n=9,3.9 \%)$ and Bacteriodes fragilis $(n=9,3.9 \%)$. When classified by species, the most common bacterial species was also E. coli ( $\mathrm{n}=113,48.9 \%)$. Streptococcus spp. $(\mathrm{n}=29,12.6 \%)$, Pseudomonas spp. $(\mathrm{n}=23,10.0 \%)$, Bacteriodes spp. $(\mathrm{n}=22,9.5 \%)$, Klebsiella spp. ( $\mathrm{n}=11,4.8 \%)$, and Enterococcus spp. $(\mathrm{n}=8,3.5 \%)$ were also common species in complicated appendicitis.

Antibiotics resistance of isolated bacteria is summarized at Table 3. There was only one case of healthcare-associated infection. All other cases were community-acquired infections. Ampicillin resistance $(n=132,57.1 \%)$ was most commonly observed. Resistance to trimethoprim/sulfamethoxazole (TMP/SMX) $(n=69$, $30.0 \%)$, cefotaxime $(n=51,22.1 \%)$, and cefazolin $(n=47,20.3 \%)$ were also relatively common; 21 cases $(9.1 \%)$ of ESBL positive, 4 cases $(1.7 \%)$ of carbapenem resistance and 1 case $(0.4 \%)$ of vancomycin resistance were observed. ESBL-producing bacteria are mostly found in E. coli $(\mathrm{n}=19,16.8 \%)$ and K. pneumonia $(\mathrm{n}=2$, 22.2\%). Vancomycin resistance was found in Enterococcus faecium, which was the only healthcare-associated infection.

E. coli reveal a variety of antibiotics resistance. Resistance to ampicillin and TMP/SMX was reported in 77 cases (68.1\%) and 47 cases $(41.6 \%)$. Resistance to cefazolin $(n=30,26.5 \%)$, cefotaxime $(n=25,22.1 \%)$, ceftazidime $(n=22,19.5 \%)$, cefepime $(n=21$, $18.6 \%)$, and gentamycin $(n=22,19.5 \%)$ were also not rare. The positive rate of ESBL was $16.8 \%$ (19 of 113). The antibiotics with the least resistance were piperacillin/tazobactam $(n=3,2.7 \%)$ and imipenem $(\mathrm{n}=2,1.8 \%)$.

$P$. aeruginosa revealed a high resistance rate to ampicillin $(\mathrm{n}=21$, $100 \%)$ and cefotaxime $(n=20,95.2 \%)$. However, resistance rates to piperacillin/tazobactam $(n=1,4.8 \%)$, ceftazidime $(n=0,0 \%)$, cefepime $(n=0,0 \%)$, ciprofloxacin $(n=1,11.1 \%)$, and imipenem $(n=0,0 \%)$ were low.

Gram-positive bacteria reveal relatively low antibiotic resistance. 


\section{Coloproctology Jung Tack Son, et al.}

Table 2. Characteristics of isolated bacteria $(n=231)$

\begin{tabular}{|c|c|}
\hline Characteristic & №. $(\%)$ \\
\hline \multicolumn{2}{|l|}{ No. of isolated bacteria } \\
\hline 1 & $121(72.9)$ \\
\hline 2 & $30(18.1)$ \\
\hline 3 & $12(7.2)$ \\
\hline 4 & $2(1.2)$ \\
\hline 6 & $1(0.6)$ \\
\hline Gram negative & 190 (82.3) \\
\hline Escherichia coli & $113(48.9)$ \\
\hline Pseudomonas aeruginosa & $21(9.1)$ \\
\hline Pseudomonas mendocina & $2(0.9)$ \\
\hline Klebsiella pneumoniae & $9(3.9)$ \\
\hline Klebsiella oxytoca & $2(0.9)$ \\
\hline Citrobacter freundii & $4(1.7)$ \\
\hline Citrobacter farmeri & $1(0.4)$ \\
\hline Citrobacter braakii & $1(0.4)$ \\
\hline Bacteroides thetaiotaomicron & $6(2.6)$ \\
\hline Bacteroides fragilis & $9(3.9)$ \\
\hline Bacteroides caccae & $3(1.3)$ \\
\hline Bacteriodes ovatus & $2(0.9)$ \\
\hline Bacteriodes uniformis & $1(0.4)$ \\
\hline Bacteriodes stercoris & $1(0.4)$ \\
\hline Comamonas testosteroni & $2(0.9)$ \\
\hline Sphingomonas paucimobilis & $2(0.9)$ \\
\hline Enterobacter cloacae & $1(0.4)$ \\
\hline Enterobacter aerogenes & $5(2.2)$ \\
\hline Burkholderia cepacia & $1(0.4)$ \\
\hline Aeromonas hydrophilia & $1(0.4)$ \\
\hline Proteus mirabilis & $1(0.4)$ \\
\hline Prevotella spp & $1(0.4)$ \\
\hline Raoultella planticola & $1(0.4)$ \\
\hline Gram positive & $41(17.7)$ \\
\hline Streptococcus anginosus & $11(4.8)$ \\
\hline Streptococcus constellatus & $10(4.3)$ \\
\hline Streptococcus pneumoniae & $1(0.4)$ \\
\hline Streptococcus intermedius & $5(2.2)$ \\
\hline Streptococcus mitis & $2(0.9)$ \\
\hline Enterococcus raffinosus & $1(0.4)$ \\
\hline Enterococcus faecium & $1(0.4)$ \\
\hline Enterococcus avium & $6(2.6)$ \\
\hline Lactococcus garvieae & $1(0.4)$ \\
\hline Peptostreptococcus micros & $1(0.4)$ \\
\hline Parvimonas micra & $2(0.9)$ \\
\hline
\end{tabular}

Only 1 Streptococcus pneumoniae and 1 E. faecium revealed resistance to antibiotics.

Bacteria isolated from patients who had complications are shown in Table 4. The results of statistical analyses for the relationships between bacterial cultures and complications are summarized in Table 5. Detection of Pseudomonas or ESBL positive cultures was not related to any complication. However, detection of Pseudomonas did affect length of hospital stay (10.5 days, $\mathrm{P}=0.042)$. Organisms with resistance to gentamycin $(\mathrm{P}=0.026)$ and TMP/SMX $(\mathrm{P}=0.002)$ were significantly correlated with organ/space SSI. Organisms with resistance to gentamycin were related to longer length of hospital stay (11.5 days, $\mathrm{P}=0.049)$. No specific organism or antibiotic resistance was related to ileus or gastroenteritis. And, patients' characteristics including sex, age, type of surgery (appendectomy or cecectomy), or ASA physical status classification were not related with any complications.

Empirical antibiotic treatment with ceftriaxone and metronidazole showed susceptibility in 174 of 231 organisms (75.3\%). Conversely, 56 of 231 (24.2\%) organisms showed resistance to empirical antibiotic treatment. Because duplicated resistant organisms were found in 1 patient, 55 patients were identified as cases of IEAT ( 55 of $173,31.8 \%$ ). These cases of IEAT revealed a significant relationship with the organ/space SSI (7 of 55, 12.7\%; $\mathrm{P}=0.005)$. However, IEAT also did not affect the rate of superficial SSI ( 2 of $55,3.6 \% ; \mathrm{P}=1.000$ ), ileus ( 2 of $55,3.6 \% ; \mathrm{P}=0.506$ ), or gastroenteritis $(0$ of $55,0 \% ; \mathrm{P}=1.000)$. The mean length of hospital stay for patients with IEAT was similar to others (9.4 days vs. 8.7 days, $\mathrm{P}=0.571$ ).

In 56 cultured organisms that reveal resistance to empiric antibiotics, the rate of cross resistance to piperacillin/tazobactam, cefepime, ciprofloxacin, imipenem, and TMP/SMX was $7.1 \%$ (4 of 56), $42.9 \%$ (24 of 56), $25.0 \%$ (14 of 56), $7.1 \%$ (4 of 56), and $67.9 \%$ (38 of 56), respectively. And ESBL-producing bacteria were identified in 21 organisms ( 21 of $56,37.5 \%$ ).

In 7 cases with organ/space SSI with IEAT diagnoses, the antibiotic regimen was changed based on the culture results. These 7 cases included 4 cases of MDR-P. aeruginosa, 1 case of ESBL-producing E. coli, 1 case of MDR-E. coli, and 1 case of vancomycinresistance $E$. faecium. A vancomycin-resistant $E$. faecium was isolated from an immunosuppressed patient, previously mentioned as healthcare-associated infection, who had been diagnosed and treated for acute myeloid leukemia, and antibiotic treatment was performed with the recommendation and approval from an infectious disease (ID) specialist. In all the other patients with community-acquired infection, the antibiotics were changed to piperacillin/tazobactam with approval by an ID specialist.

\section{DISCUSSION}

In the treatment of complicated appendicitis, the 2 main components are source control and antibiotic therapy. Source control generally includes draining the abscess or surgical removal of the 


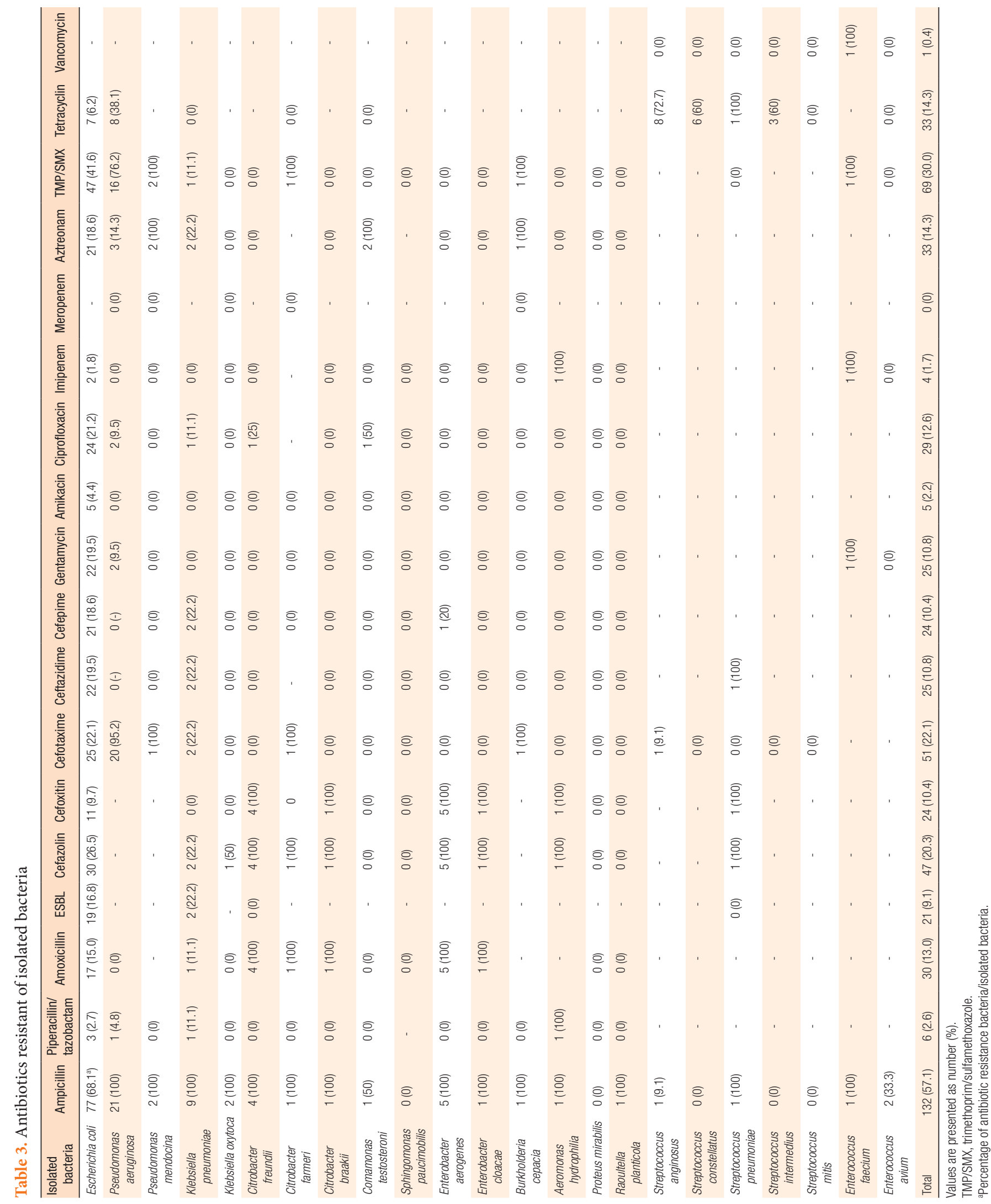




\section{Coloproctology}

Table 4. Complications and isolated bacteria

\begin{tabular}{|c|c|c|c|c|c|c|c|c|c|c|}
\hline & $\begin{array}{c}\text { Escherichia } \\
\text { coli }\end{array}$ & $\begin{array}{c}\text { Streptococcus } \\
\text { constellatus }\end{array}$ & $\begin{array}{c}\text { Streptococcus } \\
\text { anguinus }\end{array}$ & $\begin{array}{l}\text { Bacteriodes } \\
\text { fragilis }\end{array}$ & $\begin{array}{c}\text { Pseudomonas } \\
\text { aeruginosa }\end{array}$ & $\begin{array}{l}\text { Enterococcus } \\
\text { faecium }\end{array}$ & $\begin{array}{c}\text { Enterobacter } \\
\text { aerogens }\end{array}$ & $\begin{array}{c}\text { Klebsiella } \\
\text { pneumoniae }\end{array}$ & $\begin{array}{c}\text { Sphingomonas } \\
\text { paucimobilis }\end{array}$ & $\begin{array}{l}\text { No. of isolated } \\
\text { bacteria }\end{array}$ \\
\hline Superficial SSI & 6 & 2 & 2 & 1 & 1 & & & 1 & & 12 \\
\hline Organ/space SSI & 6 & & 2 & & 4 & 1 & & & & 13 \\
\hline lleus & 8 & 1 & & & & & 1 & 1 & & 11 \\
\hline Gastroenteritis & 1 & & & & & & & & 1 & 2 \\
\hline
\end{tabular}

SSI, surgical site infection.

Table 5. Statistical analysis for complication related factor

\begin{tabular}{|c|c|c|c|c|c|c|c|c|c|c|}
\hline & $\begin{array}{c}\text { Superficial } \\
\text { SSI }\end{array}$ & P-value & $\begin{array}{c}\text { Organ/space } \\
\text { SSI }\end{array}$ & P-value & lleus & P-value & Gastroenteritis & P-value & $\begin{array}{l}\text { Mean hospital } \\
\text { stay (day) }\end{array}$ & P-value \\
\hline Pseudomonas & $0(0)$ & $0.599^{\mathrm{a}}$ & $3(15.0)$ & $0.071^{\mathrm{a}}$ & $0(0)$ & $0.367^{\mathrm{a}}$ & $1(5.0)$ & $0.218^{\mathrm{a}}$ & 10.5 & $0.042^{b}$ \\
\hline ESBL positive & $1(4.8)$ & $1.000^{\mathrm{a}}$ & $1(4.8)$ & $1.000^{\mathrm{a}}$ & $2(9.5)$ & $0.626^{a}$ & $0(0)$ & $1.000^{\mathrm{a}}$ & 10.1 & $0.290^{\mathrm{b}}$ \\
\hline \multicolumn{11}{|c|}{ Antibiotics resistance } \\
\hline Ampicillin & $3(2.5)$ & $0.054^{a}$ & $9(7.4)$ & $0.059^{\mathrm{a}}$ & $7(5.8)$ & $0.736^{\mathrm{a}}$ & $0(0)$ & $0.089^{a}$ & 9.2 & $0.455^{b}$ \\
\hline Gentamycin & $0(0)$ & $0.604^{\mathrm{a}}$ & $4(16.0)$ & $0.026^{\mathrm{a}}$ & $3(12.0)$ & $0.200^{\mathrm{a}}$ & $0(0)$ & $1.000^{\mathrm{a}}$ & 11.5 & $0.049^{b}$ \\
\hline TMP/SMX & $1(1.5)$ & $0.157^{\mathrm{a}}$ & $8(12.1)$ & $0.002^{\mathrm{a}}$ & $4(6.1)$ & $1.000^{\mathrm{a}}$ & $0(0)$ & $0.525^{\mathrm{a}}$ & 9.7 & $0.236^{b}$ \\
\hline Vancomycin & $0(0)$ & $1.000^{\mathrm{a}}$ & $1(33.3)$ & $0.149^{\mathrm{a}}$ & $0(0)$ & $1.000^{\mathrm{a}}$ & $0(0)$ & $1.000^{\mathrm{a}}$ & 15.7 & $0.966^{\circ}$ \\
\hline Imipenem & $0(0)$ & $1.000^{\mathrm{a}}$ & $1(25.0)$ & $0.194^{\mathrm{a}}$ & $0(0)$ & $1.000^{\mathrm{a}}$ & $0(0)$ & $1.000^{\mathrm{a}}$ & 14.8 & $0.268^{\circ}$ \\
\hline IEAT & $2(3.6)$ & $1.000^{\mathrm{a}}$ & $7(12.7)$ & $0.005^{\mathrm{a}}$ & $2(3.6)$ & $0.506^{\mathrm{a}}$ & $0(0)$ & $1.000^{\mathrm{a}}$ & 9.4 & $0.571^{\mathrm{b}}$ \\
\hline
\end{tabular}

Values are presented as number (\%).

ESBL, extended-spectrum beta lactamase; TMP/SMX, trimethoprim/sulfamethoxazole; IEAT, inappropriate empiric antibiotic treatment,

${ }^{a}$ Fisher exact test. ${ }^{b}$ Student t-test. 'Mann-Whitney test.

appendix. However, recently, these classical ideas have been changed. The new WSES Jerusalem guidelines suggest nonoperative treatment with antibiotics could be effective in selected patients with uncomplicated appendicitis [5]. In the same guideline, nonoperative management is also suggested as a reasonable first treatment for appendicitis with phlegmon or abscess [5]. In this era of changing the concept of traditional treatment, effective antimicrobial treatment is becoming more important in the treatment of appendicitis.

However, the initial choice of antibiotics is always empiric because patients need immediate treatment, while bacterial growth to obtain results of culture and antibiotic susceptibility needs about 24-72 hours. Therefore, recent bacteriologic data are needed clinically to choose the appropriate antibiotics. Moreover, emerging MDR bacteria makes selection of empiric antibiotics more difficult.

Previous studies have reported the most common isolated bacteria in appendicitis to be E. coli. Streptococcus spp., Enterococcus spp., and $P$. aeruginosa are also commonly observed. However, the proportion of each bacterial type and resistance rate vary [10-13]. The study for Monitoring Antimicrobial Resistance Trends (SMART) trial is the only large study that includes data from 122 hospitals in 39 countries focusing on epidemiology and antibiotic suscepti- bility in acute appendicitis. This study revealed the highest prevalence of ESBL-producing E. coli (16.6\%) in the Asia-Pacific region. All species studied for the test were quite susceptible to carbapenems [7].

In this study, E. coli $(\mathrm{n}=113,48.9 \%)$, Streptococcus spp. $(\mathrm{n}=29$, $12.6 \%)$, Pseudomonas spp. $(\mathrm{n}=23,10.0 \%)$, Bacteriodes spp. $(\mathrm{n}=22$, $9.5 \%)$, Klebsiella spp. $(\mathrm{n}=11,4.8 \%)$, and Enterococcus spp. $(\mathrm{n}=8$, $3.5 \%)$ are common. This result shows relatively lower rate of $E$. coli and higher rate of Pseudomonas spp. than previous studies [11, $12]$. The rate of ESBL-producing E. coli was revealed as $16.8 \%$. The antibiotic resistance rate throughout all cultured organisms to cefoxitin, cefotaxime, ceftazidime, and ciprofloxacin were $10.4 \%$, $22.1 \%, 10.8 \%$, and $12.6 \%$, respectively. E. coli revealed respective resistance rate to these antibiotics as $9.7 \%, 22.1 \%, 19.5 \%$, and $21.2 \%$. In SMART trial, resistance rate of $E$. coli to cefoxitin, ceftriaxone, ceftazidime, and ciprofloxacin were $9 \%, 10.1 \%, 10.1 \%$, and $22.0 \%$, respectively. And ESBL-producing E. coli was detected in $8.9 \%$ worldwide. The result of this study reveals that antibiotic resistance rate in Koreans is higher than other countries. And the rate of ESBL-producing E. coli in this study is similar to the data of Asia-Pacific region in SMART trial (16.6\%) [7]. Pseudomonas aeruginosa revealed very high resistance to cefotaxime, $95.2 \%$. Moreover, 230 of 231 isolated bacteria were community-acquired 
infections. These results show that the isolated bacteria in complicated appendicitis have a notably high rate of MDR, even in patients with community-acquired infection.

Current guidelines from SIS-IDSA and WSES recommend a several antibiotics regimen for community-acquired complicated appendicitis [3-5]. Among these regimens, the combination of ceftriaxone and metronidazole has been reported as appropriate in comparison with the past regimen, with a rate of IEAT reported at $4.1 \%$ ( 2 of 49 ) $[14,15]$. In this study, the rate of IEAT was $31.2 \%$ (54 of 173) using the same antibiotic regimen. This quite high rate of IEAT suggests that some countries have a higher risk of IEAT, like Korea, meet the moment to consider changing the empiric antibiotic regimen for specific high-risk patients. For this, well-designed, multicenter studies are needed.

In this study, detection of Pseudomonas and resistance to gentamycin were related with longer length of hospital stay $(\mathrm{P}=0.042$ and $\mathrm{P}=0.049$, respectively). Resistance to gentamycin and TMPSMX were related with a higher rate of organ/space SSI $(\mathrm{P}=0.026$ and $\mathrm{P}=0.002$, respectively). The mechanism of this relationship was not proven in this study; additional study is needed to prove this clearly. Length of hospital stay in IEAT patients was not statistically different from other, non-IEAT patients, most likely because there are so many other factors that can lead to longer hospital stay, such as ileus.

Recently, several studies have claimed that intraoperative culture is not needed in acute appendicitis. These studies argue that intraoperative culture did not influence clinical outcome in patients undergoing appendectomy. The reason for their argument is that the rate of positive culture is relatively low in most cases and cases needed to change antibiotics are rare because cultured organisms are mostly susceptible to empiric antibiotics [10, 16-19]. However, all studies questioning the use of intraperitoneal swabs were open, nonrandomized, or retrospective studies with incompletely matched control groups and nonstandardized swab collection techniques; consequently, they lacked power to inform surgical practice [20]. Our study is also nonrandomized and retrospective; therefore, we also cannot definitively show evidence that a specific organism affected morbidity or length of hospital stay of patients. However, our study reveals that IEAT increases organ/space SSI (7 of 54). Seven patients with IEAT needed to change antibiotics according to the results of culture, and they all recovered without any additional problems.

Bacterial culture is an inexpensive, simple test that does not harm the patient. Therefore, routine intraoperative culture is necessary, at least in complicated appendicitis, although the majority of the patients may not need it.

In the present study, the second choice of antibiotics in all community-acquired infection was piperacillin/tazobactam according to the antibiotic susceptibility tests. Even in patients with ESBLproducing bacteria, if piperacillin/tazobactam was revealed as effective in vitro, it was used. Although carbapenem has been considered the drug of choice for treating ESBL-producing bacteria, some authors report piperacillin/tazobactam is a useful alternative and not inferior to carbapenem [21-23]. Since piperacillin/tazobactam and carbapenem reveal high susceptibilities $(97.4 \%$ and $98.3 \%$, respectively) in this study, these 2 antibiotics could be selected in suspecting resistance of empirical antibiotics, even if the result of culture is not available.

This report includes relatively the small number of patients, because the bacterial culture was not routinely performed intraoperatively. In our institution, bacterial culture was selectively performed by the discretion of each surgeon and only 1 surgeon routinely performed intraoperative bacterial culture in most cases with complicated appendicitis.

The methods of culture were different depending on the type of complicated appendicitis in this study. When suppurative fluid or abscess is present, these are collected in culture tube. In cases of gangrenous appendicitis without free perforation, swabbing of appendiceal lumen was performed for culture. Up to now, there has not been a standard method of culture for complicated appendicitis. Especially, the culture method of appendiceal swab is not standardized [20]. Therefore, further studies are needed to develop an approved standard method of routine intraoperative culture in patients with complicated appendicitis.

The rate of IEAT was $31.8 \%$ in complicated appendicitis. IEAT did not lead to adverse clinical outcomes in the majority of patients. This means that proper source control still plays a more important role in treatment of complicated appendicitis. Nevertheless, IEAT for complicated appendicitis may lead to increased rates of organ/space SSI.

In conclusion, the rate of antibiotic resistance was high in community-acquired complicated appendicitis in Koreans. Routine intraoperative culture in patients with complicated appendicitis may provide information about an appropriate antibiotic regimen when current empiric antibiotics treatment fails.

\section{CONFLICT OF INTEREST}

No potential conflict of interest relevant to this article was reported.

\section{REFERENCES}

1. Addiss DG, Shaffer N, Fowler BS, Tauxe RV. The epidemiology of appendicitis and appendectomy in the United States. Am J Epidemiol 1990;132:910-25.

2. Sartelli M, Catena F, Coccolini F, Pinna AD. Antimicrobial management of intra-abdominal infections: literature's guidelines. World J Gastroenterol 2012;18:865-71.

3. Solomkin JS, Mazuski JE, Bradley JS, Rodvold KA, Goldstein EJ, Baron EJ, et al. Diagnosis and management of complicated intraabdominal infection in adults and children: guidelines by the Surgical Infection Society and the Infectious Diseases Society of America. Clin Infect Dis 2010;50:133-64. 
4. Sartelli M, Chichom-Mefire A, Labricciosa FM, Hardcastle T, Abu-Zidan FM, Adesunkanmi AK, et al. The management of intra-abdominal infections from a global perspective: 2017 WSES guidelines for management of intra-abdominal infections. World J Emerg Surg 2017;12:29.

5. Di Saverio S, Birindelli A, Kelly MD, Catena F, Weber DG, Sartelli $\mathrm{M}$, et al. WSES Jerusalem guidelines for diagnosis and treatment of acute appendicitis. World J Emerg Surg 2016;11:34.

6. Reinisch A, Malkomes P, Habbe N, Bechstein WO, Liese J. Bad bacteria in acute appendicitis: rare but relevant. Int J Colorectal Dis 2017;32:1303-11.

7. Bochicchio GV, Baquero F, Hsueh PR, Paterson DL, Rossi F, Snyder TA, et al. In vitro susceptibilities of Escherichia coli isolated from patients with intra-abdominal infections worldwide in 2002-2004: results from SMART (Study for Monitoring Antimicrobial Resistance Trends). Surg Infect (Larchmt) 2006;7:537-45.

8. Cardoso T, Almeida M, Friedman ND, Aragão I, Costa-Pereira A, Sarmento AE, et al. Classification of healthcare-associated infection: a systematic review 10 years after the first proposal. BMC Med 2014;12:40.

9. Kariv G, Paul M, Shani V, Muchtar E, Leibovici L. Benchmarking inappropriate empirical antibiotic treatment. Clin Microbiol Infect 2013;19:629-33.

10. Akingboye AA, Davies B, Tien T. Pus Samples in complicated appendicitis: an important investigation or a waste of resources: a prospective cohort study. Scand J Surg 2019;108:55-60.

11. Chen CY, Chen YC, Pu HN, Tsai CH, Chen WT, Lin CH. Bacteriology of acute appendicitis and its implication for the use of prophylactic antibiotics. Surg Infect (Larchmt) 2012;13:383-90.

12. Coccolini F, D’Amico G, Sartelli M, Catena F, Montori G, Ceresoli $\mathrm{M}$, et al. Antibiotic resistance evaluation and clinical analysis of acute appendicitis; report of 1431 consecutive worldwide patients: A cohort study. Int J Surg 2016;26:6-11.

13. Jeon HG, Ju HU, Kim GY, Jeong J, Kim MH, Jun JB. Bacteriology and changes in antibiotic susceptibility in adults with community-acquired perforated appendicitis. PLoS One 2014;9:e111144.
14. St Peter SD, Tsao K, Spilde TL, Holcomb GW 3rd, Sharp SW, Murphy JP, et al. Single daily dosing ceftriaxone and metronidazole vs standard triple antibiotic regimen for perforated appendicitis in children: a prospective randomized trial. J Pediatr Surg 2008;43:981-5.

15. Tan A, Rouse M, Kew N, Qin S, La Paglia D, Pham T. The appropriateness of ceftriaxone and metronidazole as empirical therapy in managing complicated intra-abdominal infection-experience from Western Health, Australia. PeerJ 2018;6:e5383.

16. Gladman MA, Knowles CH, Gladman LJ, Payne JG. Intra-operative culture in appendicitis: traditional practice challenged. Ann R Coll Surg Engl 2004;86:196-201.

17. Foo FJ, Beckingham IJ, Ahmed I. Intra-operative culture swabs in acute appendicitis: a waste of resources. Surgeon 2008;6:278-81.

18. Celik A, Ergün O, Ozcan C, Aldemir H, Balik E. Is it justified to obtain routine peritoneal fluid cultures during appendectomy in children? Pediatr Surg Int 2003;19:632-4.

19. Kenig J, Richter P. The need for culture swabs in laparoscopically treated appendicitis. Wideochir Inne Tech Maloinwazyjne 2013;8: 310-4.

20. Davies HO, Alkhamesi NA, Dawson PM. Peritoneal fluid culture in appendicitis: review in changing times. Int J Surg 2010;8:426-9.

21. Gavin PJ, Suseno MT, Thomson RB Jr, Gaydos JM, Pierson CL, Halstead DC, et al. Clinical correlation of the CLSI susceptibility breakpoint for piperacillin- tazobactam against extended-spectrum-beta-lactamase-producing Escherichia coli and Klebsiella species. Antimicrob Agents Chemother 2006;50:2244-7.

22. Kang CI, Park SY, Chung DR, Peck KR, Song JH. Piperacillintazobactam as an initial empirical therapy of bacteremia caused by extended-spectrum $\beta$-lactamase-producing Escherichia coli and Klebsiella pneumoniae. J Infect 2012;64:533-4.

23. Peterson LR. Antibiotic policy and prescribing strategies for therapy of extended-spectrum beta-lactamase-producing Enterobacteriaceae: the role of piperacillin-tazobactam. Clin Microbiol Infect 2008;14 Suppl 1:181-4. 